\title{
A New Prototype of Smart Wearable Monitoring System Solution for Alzheimer's Patients
}

\author{
Bassam Al-Naami (iD \\ Hamza Abu Owida ${ }^{2}$ \\ Mohammed Abu Mallouh ${ }^{3}$ \\ Feras Al-Naimat ${ }^{2}$ \\ Moh'd Agha ${ }^{2}$ \\ Abdel-Razzak Al- \\ Hinnawi (iD ${ }^{4}$ \\ 'Department of Biomedical Engineering, \\ The Hashemite University, Zarqa, Jordan; \\ ${ }^{2}$ Department of Medical Engineering, Al- \\ Ahliyya Amman University, Amman, \\ Jordan; ${ }^{3}$ Department of Mechatronics \\ Engineering, The Hashemite University, \\ Zarqa, Jordan; ${ }^{4}$ Faculty of Sciences, Al- \\ Isra University, Amman, Jordan
}

\begin{abstract}
Purpose: The daily life management of patients with Alzheimer's disease (AD) constitutes a significant and rapidly expanding health-care responsibility. In this study, an innovative prototype of a wireless-sensing smart wearable medical device (SWMD) is proposed as a multi-functions solution for Alzheimer patients. The SWMD is aimed to assemble three main biomedical engineering advances: 1) use of a Wi-Fi microcontroller, 2) simultaneous monitoring of a set of vital biomarkers, and 3 ) cautions of fall down conditions, in addition to GPS location indicator.
\end{abstract}

Methods: The SWMD employs a Wi-Fi controller that is incorporated with electronic circuits to monitor three vital signals (temperature, heart rate, and oxygen saturation), fall down conditions in three directions (X, Y, and Z axis), and GPS location. The SWMD was connected to the Firebase Service (database hosted on the Internet Cloud). The proposed device was tested on 13 normal volunteers. The left side, right side, forward, and backward fall down conditions were assessed. The prototype's functions during daily activity such as rising hand, sitting down or standing up, and walking conditions were also assessed.

Results: The three assembled functions were all successfully incorporated to build the SWDM device as a suggested solution offering real-time alerts during daily activity to AD patients. The Bland-Altman statistical test showed no significant difference ( $p$-value $>0.05$ ) between the SWMD biomarkers' acquisition and the reference methods. The gyro/accelerator sensor yielded $93 \%$ sensitivity in fall down detection and $95 \%$ specificity during daily activities. The GPS yielded correct positioning of the SWDM holder, while the internet cloud allowed saving and managing all vital biomarkers daily.

Conclusion: The SWMD is a possible solution for daily life support for AD patients. It incorporates three functions in one single device, GPS location indicator, monitoring set of biomarkers, and fall down alert, which are all controlled via a Wi-Fi micro controller on-line connected to Internet Cloud. It successfully would allow the management of the daily records as well as the real-time alerts to remote persons.

Keywords: Alzheimer's disease, smart wearable medical device, fall down alert, Internet Cloud access, health care aids, Wi-Fi micro controller

\section{Introduction}

Alzheimer's disease (AD) is still incurable. ${ }^{1}$ The emotional and psychological repercussions are distressing for both patients and relatives. It has been reported that the progression of AD takes 12 years in three stages. ${ }^{1,2}$ The first stage, which lasts one to three years, starts with signs of aberrant biomarkers and recurring mild to moderate cognitive problems. In the second stage (three to ten years), the AD patients suffer from temporal memory loss, repetitive moderate cognitive impairment, and the presence of pathophysiological biomarkers. Eventually, the third
Correspondence: Bassam Al-Naami Department of Biomedical Engineering, Faculty of Engineering, The Hashemite University, PO Box 330127, Zarqa, I3133, Jordan

Fax +962 53826348

Email b.naami@hu.edu.jo 


\section{Graphical Abstract}

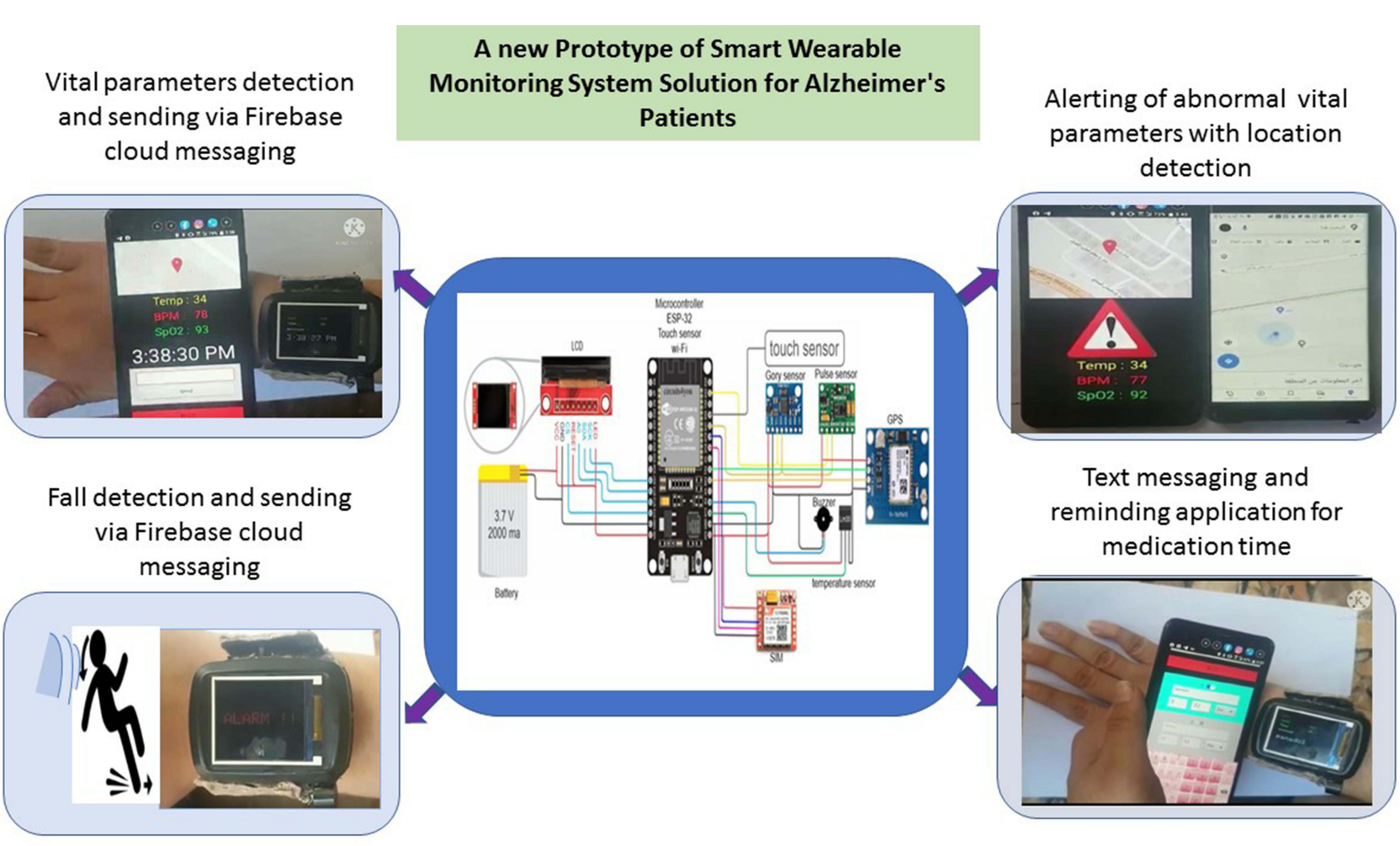

stage, which is also called dementia, persists for eight to twelve years. Regrettably, AD patients will likely suffer apparent memory loss, evident cognitive deterioration, and frequent unexpected aberrant biomarkers. ${ }^{1-3}$ Further research suggests that cognitive, behavioural, sensory, and motor changes may begin to appear prior to the appearance of clinical symptoms of AD several years in advance. ${ }^{4,5}$ Unfortunately, AD's prevalence is expected to influence around 74.7 million people by the year $2030 .^{2,6,7}$

Therefore, AD patients' life-support devices are in demand and it is an ongoing research topic. ${ }^{1,2,8}$ Biomedical engineering (BME) health-care technologies for $\mathrm{AD}$ patients are gradually making their way into our daily lives. ${ }^{9}$ Currently, BME technologies offer solutions of AD's medical care support devices, allowing AD patients to conduct their various daily routine tasks safely. ${ }^{6}$ One of which is the voice assistant devices, at which voice recordings help AD's patients in their daily life by playing sound notifications of time of medication, appointments, and date and time; these are simple sound "reminder" devices. ${ }^{10}$ Other devices employed the Internet Wi-Fi technology with geographical position system (GPS) application; these are telemetry medical assistance devices (TMAD). ${ }^{11-15}$ They are currently being used in homes and nursing homes to manage people's health yielding considerable degree of reliability. ${ }^{11,12}$ A GPS tracker, which is sealed under the insole of the shoes (eg, Smartsole), was suggested. ${ }^{14}$ It monitors the location of someone who wander such as AD patients. The GPS was also utilized in a smart-watch with life-saving capabilities. ${ }^{13}$ In addition to tracking the current location of caregivers, the smart watch allows monitoring heart rate, receiving text and voice messages along with vibration alerts. They are equipped with a help push button (ie, an emergency SOS button). However, these GPS devices face some difficulties. $^{11-14}$ The antenna, which is mounted on AD patients' bodies, may provoke some complexity and discomfort. They require specialized sensors and vendor-specific software, resulting in high price expectancy. The most important drawback is the fact that these GPS devices have no fall alert sensors and it may be impractical for people with hand dexterity.

On the other hand, utilizing deep learning and face recognition has been also suggested to improve the life quality of $\mathrm{AD}$ patients. ${ }^{16,17}$ It was designed as a "smart 


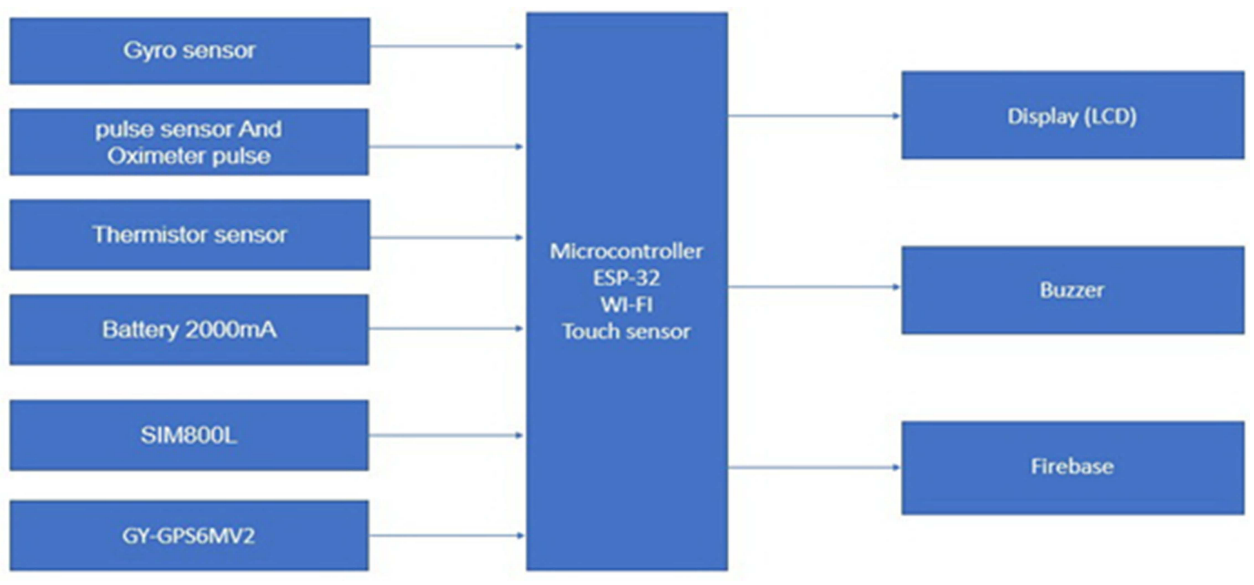

Figure I The block diagram of the proposed SWMD prototype to aid the AD patients.

cap" that can assist $\mathrm{AD}$ patients in detecting persons they know (eg, family) or even those who might harm them was investigated. ${ }^{16}$ It is a high price technology restricted to machine learning know how. Although it helps in recognizing people using previously trained model but it neither monitor vital parameters nor it can provide alarms of fall down conditions, which are frequent events for $\mathrm{AD}$ patients.

However, some researchers investigated prototypes to detect only fall down conditions. In the work by Ramachandran and Karuppiah, ${ }^{25}$ a survey of the recent work was presented. Their accuracy was in the range $79 \%$ to $100 \%$ utilizing different approaches like environmental sensing-based systems, wearable sensor-based systems and vision-based systems.

Therefore, it is expected that health-care BME technologies will keep progressing and replacing older devices with more intelligent ones, proposing solutions to unsolved problems. ${ }^{1}$ In this paper, a new prototype of multifunctional sensing smart wearable monitoring device (SWMD) was designed, built, and tested. The SWMD is worn on wrist and has three major advances over the existent devices. First, the SWMD is equipped with a fall down alert that utilizes gyro and accelerometer sensor. Second, the SWMD utilizes a Wi-Fi microcontroller with access to the Firebase Service (database hosted on the Internet Cloud) to enable health care and family members of being at real-time contact with the AD-patient. These two latter specifications, to the best of our knowledge, neither was addressed in literature nor was equipped in a the existent devices. Additionally, the third technical feature, the prototype presents a new configuration to provide simultaneous monitoring of a set of vital bio signals (ie, biomarkers) such as temperature, heart pulse, and oxygen level.

\section{Materials and Methods}

Figure 1 shows the block diagram of the proposed SWMD for AD patients. It consists of six input sensors, microcontroller, batteries and two output devices. The InvenSense MPU-6050 Gyro and accelerometer sensor is one of the six input sensors used in the proposed SWMD. Figure 2 shows the MPU-6050 that is capable of measuring acceleration along the $\mathrm{x}, \mathrm{y}$, and $\mathrm{z}$ axes and the angular velocity about the $x, y$, and $z$ axes. The fall down of the AD's patient can be detected when the MPU-6050 sensor reads up normal values for acceleration and angular velocities. The heart rate and the Oxygen saturation (SpO2) in blood are measured using MAX30100. Figure 3 shows the MAX30100, which is an integrated pulse oximetry and heart rate sensor. It consists of two LEDs, a photodetector, optimized optics, and low noise signal processing to detect pulse oximetry and heart rate signals. ${ }^{18}$ This sensor emits two wavelengths of light (red and infrared) from two LEDs. Then the reflected light is measured and processed in order to estimate two biomarkers, the pulses rate and the $\mathrm{SpO} 2$. The patient temperature is another biomarker that is measured using the Texas Instrument LM35 temperature measuring IC. This temperature sensor has an accuracy of $\pm 0.5 \mathrm{C}^{\circ}$. The fifth input signal to the SWMD comes from a GPS GPS6MV2 module. It is used to determine the latitude and longitude coordinates of the SWMD. Finally, a contact sensor is used to sense if the patient has appropriately put on the SWMD.

The outputs of the SWMD are of three types: visual output through a LCD, sound output through a buzzer, and data output through the Firebase real-time database. The LCD displays the patient's information and biomarkers. It has a screen size of 1.8 inches, and it has an up to $2 \mathrm{~GB}$ 


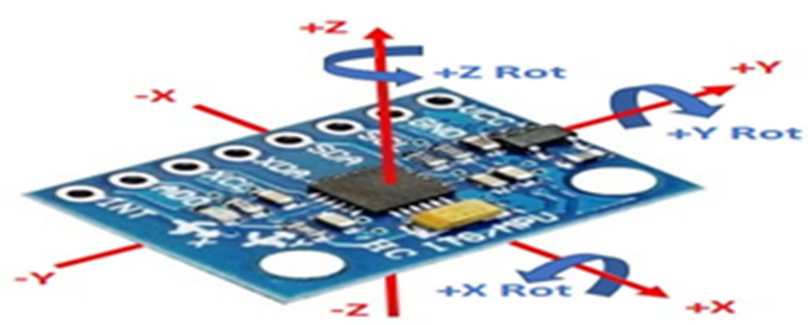

Figure 2 The gyro and accelerometer sensor module.

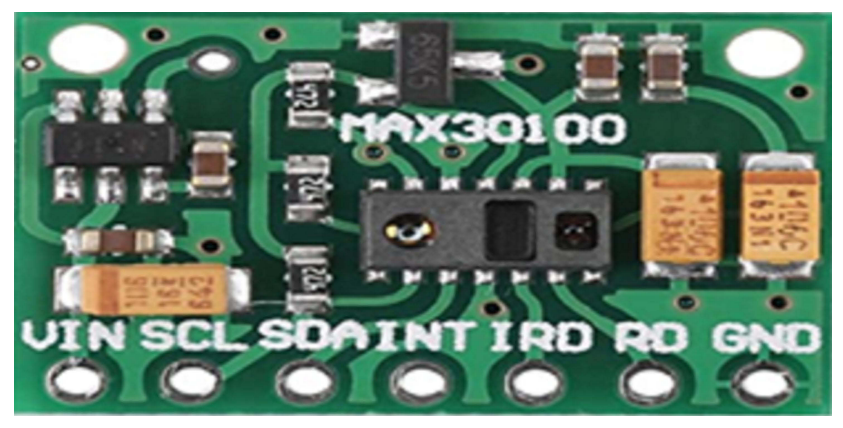

Figure 3 The MAX30I00 pulse oximeter and heart rate sensor integrated circuit.

SD card slot to store information. A buzzer is added to the system to send a sound alarm when sensors read abnormal values. This alarm brings the attention of both the patient and the surroundings, indicating emergency situation to handle. The Firebase Real-time database is a database hosted on the cloud and is used to share the SWMD's readings with others (ie, patient's doctor and relatives) through the cloud. The data is stored and synchronized in real time with each connected client. The SWMD is connected to the internet through a SIM800L module. The SWMD is powered by $3.7 \mathrm{~V}$ lithium-ion batteries with a capacity of $2000 \mathrm{mAh}$. The energy in the batteries is enough to run the device for approximately 10 hours continuously and they can be charged through a charger port.

With regard to the controller hardware, an ESP32WROOM-32 microcontroller module by ESPRESSIF is used. The microcontroller has two CPU cores that can be individually controlled, with a CPU clock up to $240 \mathrm{MHz}$ and 4 MB RAM. It also integrates a rich set of peripherals (ie, capacitive touch sensors, Hall sensors, SD card interface, Ethernet, Bluetooth, Wi-Fi). Figure 4 shows the components and connections diagram for the SWMD. All sensors were defined on the microcontroller using Arduino as a programming language. Figure $5(\mathrm{~A}-\mathrm{D})$ shows the top (A), bottom (B), right and left sides (C and D) of the SWMD after assembly.

Figure 6 shows the operating flow chart for the SWMD. First, the SWMD is turned on. Then, it starts searching and connecting to a Wi-Fi network. If it does not connect to a Wi-Fi network, the SWMD will be turned off. If it is connected to a Wi-Fi network, then the touch sensor will be checked to make sure that the SWMD is worn properly by the user. After confirming proper internet connecting and wearing of the SWMD, sensors will start operating and sending measurements to the LCD and Firebase through the microcontroller. The SWMD is set to measure $\mathrm{AD}$ patient's temperature, pulse rate, $\mathrm{SpO}$, acceleration, angular velocity and GPS location every 10 seconds and share the readings through the Firebase with medical care providers. If any of the measured values goes beyond normal readings, the SWMD will turn the alarm

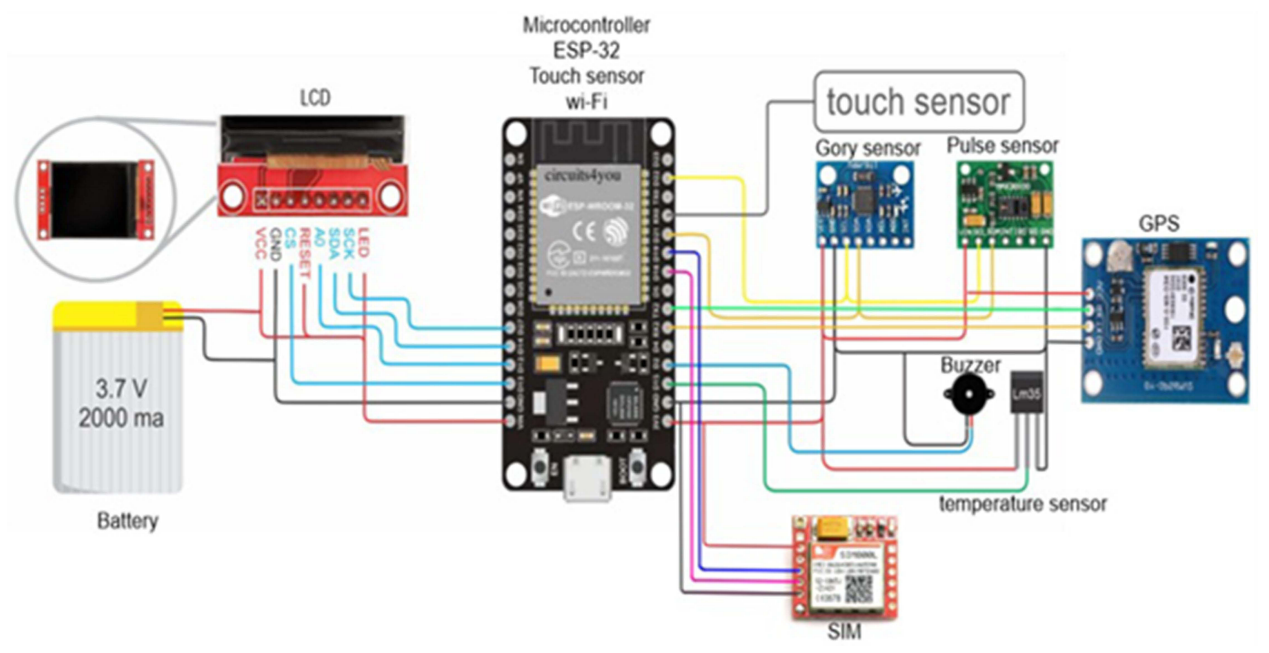

Figure 4 Components and connections for the smart wearable monitoring device. 
A

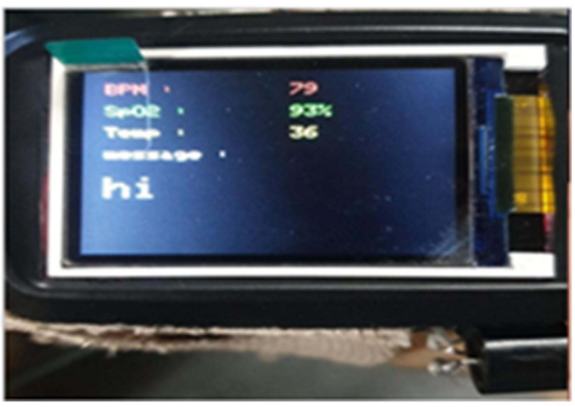

C

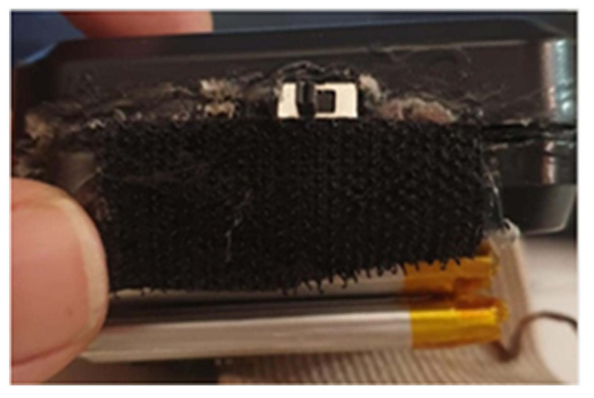

B

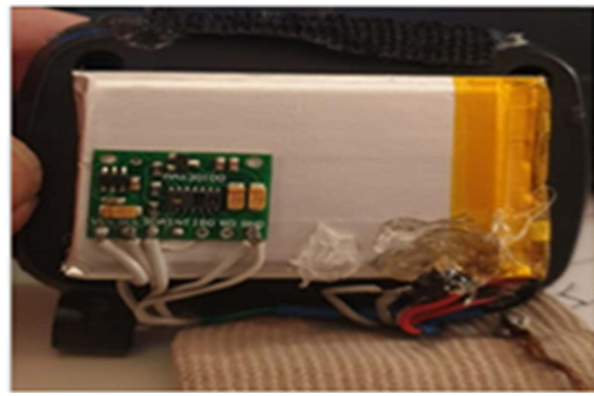

D

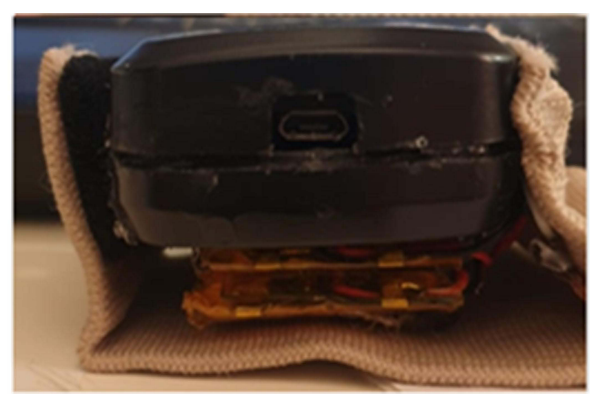

Figure 5 The SWMD after assembly. (A) Top view, (B) Bottom view, (C) Right side view and (D) Left side view.

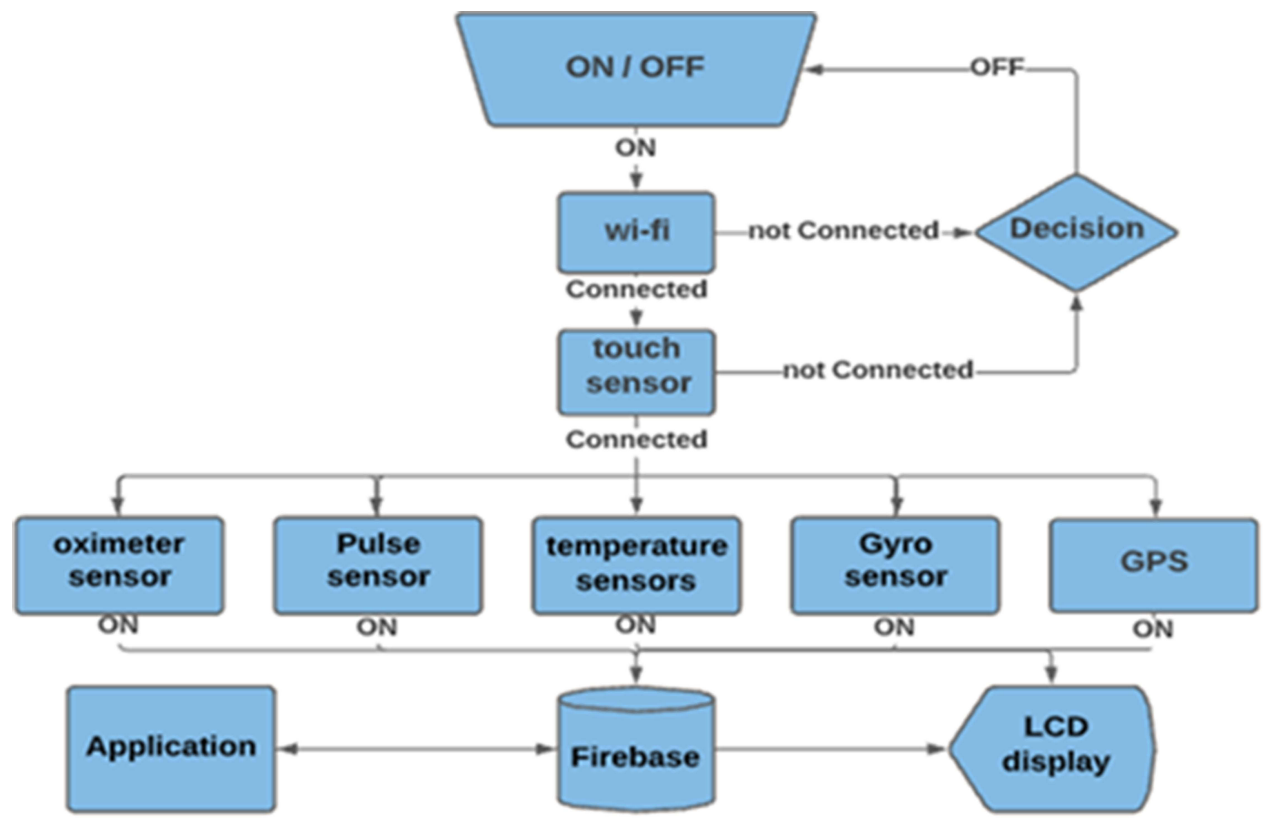

Figure 6 The operating flow chart for the SWMD.

buzzer on and will send a warning message to relatives or medical care provider through the internet cloud.

The proposed SWMD was tested on 13 normal volunteers aged 22 to 33 years. The results of these tests were presented using descriptive statistics. One-way ANOVA was used in the analysis, followed by a Tukey's post-test; significance was approved at $\mathrm{p}$-value $<0.05$. In addition, the Bland Altman approach was employed to assess the agreement between the proposed and existing reference measurements. ${ }^{19}$ The study was approved by the research ethical committee at Al-Ahliyya Amman University and was conducted in accordance with declaration of Helsinki. All volunteers (normal) assigned their informed consent prior to participating in the study. It was difficult to have the consent from AD patients. Supervising patients such as AD patients require medical precautions, which could not be achieved by 
scientist (the authors are biomedical engineers). In our institution, it is against research ethics investigating experiments on $\mathrm{AD}$ people with possible risk to fall down, or asking them to attempt fall down experiment without the intervention of medical physicians.

\section{Results}

The block diagram, electronic components, and the flow chart of the proposed SWMD (ie, Figures 1, 4 and 6) were successfully assembled, and a prototype of the wearable device is constructed as shown in Figure 5. The Supplementary Video shows a demonstration of the SWMD operation. The video demonstrates the different outputs and alerts yielding from the SWMD.

The 13 participants were asked to wear the SWMD (Figure 5) on their wrists. Although the participants have no medical conditions, these tests will allow checking the various functionality of the proposed SWMD. Temperature, pulse rate, and $\mathrm{SpO} 2$ for each volunteer were recorded and then compared with the same parameters recorded by standard medical devices. These measurements were expressed as mean \pm standard deviation (SD) for statistical analysis. Table 1 shows the results. One-way ANOVA was used to determine statistical significance, followed by a Tukey's post-test. Significance was confirmed at $p$-value $<0.05$. The mean $\pm \mathrm{SD}$ for reference temperature measurements was $35.2 \pm 0.77 \mathrm{C}^{\circ}$, while it was $35.3 \pm 0.84 \mathrm{C}^{\circ}$ for the temperature measurements from the proposed SWMD. The measurements of pulse rate yielded $80.7 \pm 5.6 \mathrm{BPM}$ and $80.4 \pm$ 6.5 BPM for reference and the SWMD, respectively. The measurements of oxygen concentration (ie, SpO2) yielded $95 \% \pm 0.022$ and $94 \% \pm 0.017$ for reference and the SWMD, respectively. The results indicated there is no statistically significant difference $(p$-value $<0.05)$ between the references testing methods and the proposed SWMD.

The Bland Altman statistical test was implemented to evaluate the agreement between the measurements from the proposed SWMD and standard medical devices. The arithmetic difference between each measurement by the SWMD and the standard device was calculated. The mean and the SD of differences were inferred. Table 2 shows the results, illustrating the mean value of differences and the mean value $\pm 1.96 \mathrm{SD}$. The upper and lower difference limits of the three biomarkers reference measurements of temperature, pulse rate, and oxygen concentration were 0.71 to $-0.51 \mathrm{C}^{\circ}, 6.24$ to -5.47 BPM, and $4.18 \%$ to $-5.18 \%$, respectively.

Figure 7 shows the Bland Altman diagram. It shows that all measurements from the SWMD are clustered around the mean \pm 1.96 SD. Since the differences between the SWMD's measurements and the standard medical devices' measurements are within the mean $\pm 1.96 \mathrm{SD}$ and are not clinically significant, two measurement methods may be used interchangeably. Moreover, the mean values for all characteristic are close to zero, implying that the developed device's measurements are reasonably consistent with those obtained with standard medical devices. As a result, the proposed SWMD can provide correct biomarkers' measurements as the standard methods.

The GPS in the SWMD determines the global position of the person wearing the device. It is tested and compared with the same position results optioned from a smart phone. Table 3 lists GPS for three different locations using both the SWMD and the smart phone. One notes that results with the SWMD and the smart phone are very close.

The emergency patient's falls down is detected through the gyro/accelerometer sensor (ie, MPU-6050). This sensor provides readings of the accelerations and angular

Table I Results of Biomarkers from Proposed SWMD and References

\begin{tabular}{|l|c|c|c|}
\hline & Mean \pm SD Reference Tests & Mean \pm SD SWMD Tests & ANOVA Test ( $p$-value) \\
\hline Pulse sensor (BPM) & $80.7 \pm 5.6$ & $80.4 \pm 6.5$ & 0.87 \\
Oxygen pulse (\%) & $95 \pm 0.022$ & $94 \pm 0.017$ & 0.31 \\
Temperature sensor $\left({ }^{\circ} \mathrm{C}\right)$ & $35.2 \pm 0.77$ & $35.3 \pm 0.84$ & 0.76 \\
\hline
\end{tabular}

Table 2 Differences in Results of Biomarkers from SWMD and References

\begin{tabular}{|l|c|c|c|c|}
\hline & Mean of Differences & SD of Differences & Mean +I.96 SD & Mean -I.96 SD \\
\hline Heart Beat sensor (BPM) & 0.39 & 2.99 & 6.24 & -5.47 \\
Oxygen Saturation $(\%)$ & -0.50 & 2.39 & 4.18 & -5.18 \\
Temperature sensor $\left({ }^{\circ} \mathrm{C}\right)$ & +0.10 & 0.31 & 0.71 & -0.51 \\
\hline
\end{tabular}



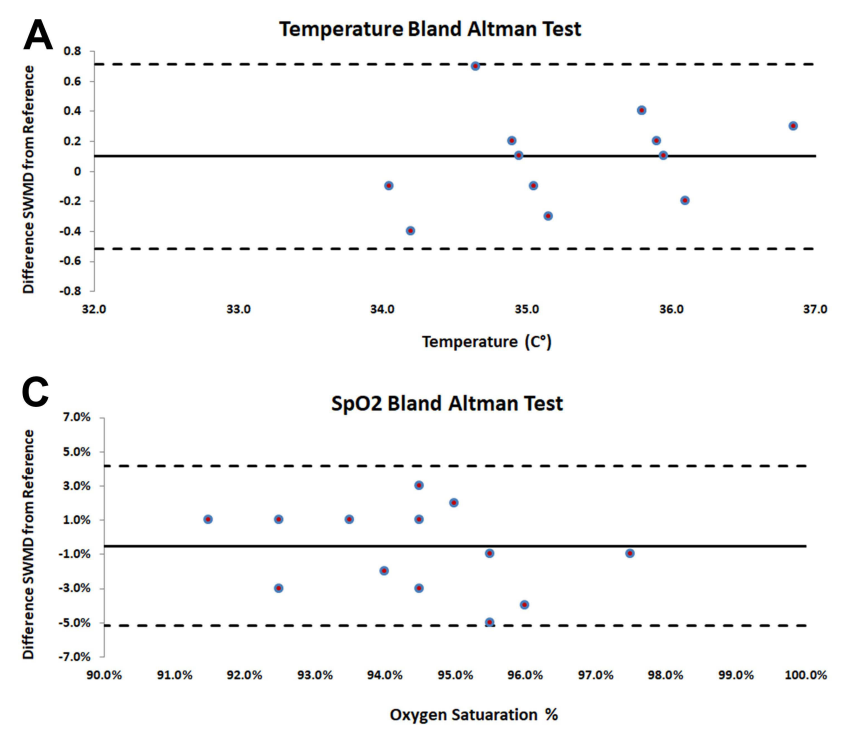

Figure 7 Bland-Altman plot of SWMD vs references readings: $(\mathbf{A})$ temperature test

velocities in the $\mathrm{x}, \mathrm{y}$, and $\mathrm{z}$ axes. These measurements are incessantly monitored through the Wi-Fi microcontroller and when up normal readings are detected the fall down alarm is turned on. The fall-down alarms can be automatically sent to remote persons such as caregivers and family members through the internet cloud. The thresholds of losing equilibrium in $\mathrm{x}, \mathrm{y}$, and $\mathrm{z}$ axes can be adjusted by the microcontroller. Supplementary Video shows an example of how the buzzer goes on in case of losing equilibrium. For this experiment, 10 out of the 13 volunteers underwent 4 tests to assess fall down alert in one of four different directions: forward, backward, left side, and right side fall down conditions. The volunteers were asked to repeat the test 4 times. Therefore, for each fall down direction we had 40 tests. Table 4 shows the sensitivity of correctly detecting fall down condition. On the other hand, the volunteers were also asked to experiment the prototype (SWDM) during some normal Activities of Daily Life (ADL). This test was also repeated four times for each ADL normal motion, walking, sitting down, standing up, and rising hand. Table 4 illustrates the success in avoiding false alarms (ie, specificity). The false positive

Table 3 GPS Results for Three Location Using SWMD and Smart Phone

\begin{tabular}{|l|c|c|}
\hline Location & With Smart Phone & With SWMD \\
\hline Location I & N32.024364, E35.859956 & N32.024364, E35.859960 \\
Location 2 & N32.0I785I, E35.8663I8 & N32.017864, E35.8663I4 \\
Location 3 & N32.0I7070, E35.866097 & N32.0I7065, E35.86608I \\
\hline
\end{tabular}

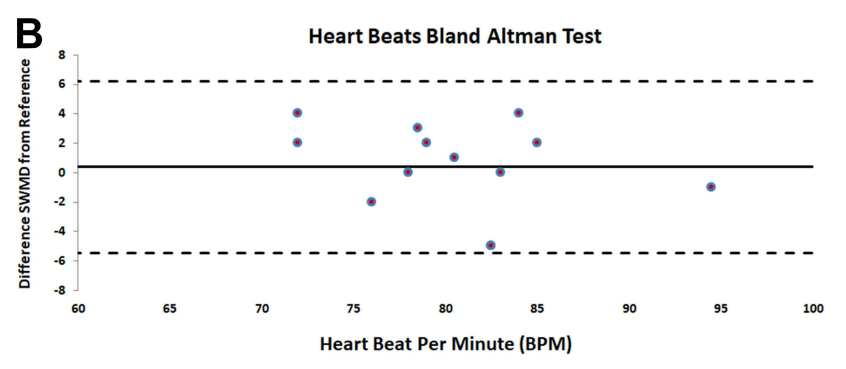

t, (B) pulse test and (C) oxygen concentration test.

(FP), true positive (TP), false negative (FN), and true negative (TN) were described according to the following conditions:

TP: represents falls down detection with alarm.

FP: represents an alarm but no fall down occurred (i.e., ADL normal motion but detected as fall down).

TN: represents no alarm during ADL normal motion.

FN: represents undetected fall down (i.e., fall down with no alarm).

Subsequently, the sensitivity (accuracy in detecting true falls) and specificity (accuracy in avoiding false alarms) were deduced according to equations:

Sensitivity $=\mathrm{TP} /(\mathrm{TP}+\mathrm{FN})$

Specificity $=\mathrm{TN} /(\mathrm{TN}+\mathrm{FP})$

\section{Discussion}

Alzheimer's disease constitutes a significant and rapidly expanding health-care responsibility. ${ }^{1}$ Although the current $\mathrm{AD}$ life assistant devices (eg, sounds recordings, ${ }^{10}$ text messages, ${ }^{1}$ GPS location, ${ }^{9,11-14,20-22}$ and face recognition devices ${ }^{16,17,23}$ ) have reaped the advances in healthcare and communication technology, there is technical incompleteness such as to assemble the evaluation of several biomarkers sensing with generations of alarms in case of fall down, which is crucial in AD daily life. ${ }^{1}$

This paper presented a prototype of a smart wearable life-support device with four innovative aspects that concern both the AD's patients and the care provider. First, it suggested a new prototype capable of simultaneously monitoring and providing alerts for three biomarkers, the 
Table 4 Results of Experiments Using the Fall Detection Sensor for 7 Different Sudden Motions. Motion Description

\begin{tabular}{|c|c|c|c|c|c|c|c|}
\hline & & TP & $\mathbf{F N}$ & $\mathbf{F P}$ & $\mathbf{T N}$ & Specificity (\%) & Sensitivity (\%) \\
\hline \multirow[t]{4}{*}{ Fall Down } & Forward fall & 38 & 2 & 0 & 0 & NA & 95.0 \\
\hline & Backward & 37 & 3 & 0 & 0 & NA & 92.5 \\
\hline & Left side fall & 37 & 3 & 0 & 0 & NA & 92.5 \\
\hline & Right side fall & 38 & 2 & 0 & 0 & NA & 95.0 \\
\hline \multirow[t]{3}{*}{$A D L$} & Walking & 0 & 0 & I & 39 & 97.5 & NA \\
\hline & Sitting down or standing up & 0 & 0 & 0 & 40 & 100.0 & NA \\
\hline & Rising hand & 0 & 0 & 5 & 35 & 87.5 & NA \\
\hline Average & & 150 & 10 & 6 & 114 & 95.0 & 93.7 \\
\hline
\end{tabular}

Abbreviation: NA, not applicable.

temperature, pulse rate, and $\mathrm{SpO} 2$. Second, but a major progress, it also proposed a solution for providing alerts in case the $\mathrm{AD}$ patients suddenly encounters any lack of equilibrium conditions due to scramble, tumble, or fall down. Third, the system can save all AD patients' daily information in the internet cloud. Fourth, all information in the SWMD can be monitored from a remote medical care observer, including the case that the AD patient is suddenly to fall down. A GPS service, indicating the location of the AD patient, is also available.

In terms of monitoring $\mathrm{AD}$ patient's bio signals, the proposed SWMD employed a well-known commercial sensor of temperature, pulse rate, and $\mathrm{SpO} 2$. The measurements on 13 participants showed that there was no significant statistical difference between calculations using the SWMD (Figures 1, 4-6) and the standard methods. Figure 7 shows the Bland Altman plot. It is statistical plot provides two key metrics, bias and range of agreement, which are used to assess the precision of a model or prototype. $^{18,19,24}$ The plots in Figure 7 shows that the measured values in SWMD were within mean \pm 1.96 standard deviations and thus were not clinically significant. Furthermore, the mean difference values for all three biomarkers are near zero, indicating that the measurements of the developed device are reasonably consistent with those of the reference testing methods. As a result, the proposed smart system, the SWMD, can be considered as a reliable substitute for current standard measurements.

In terms of fall alerts, cautions are turned on when the microcontroller detects an up normal reading from the gyro/ accelerometer sensor, as shown in the Supplementary

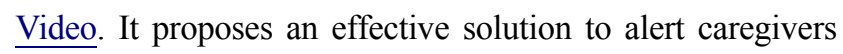
in case of fall down conditions, which has not been solved in the other AD's life support devices. It presents a significant improvement that would allow immediate action upon fall down emergency. Table 4 shows that the prototype could achieve preliminary $93.7 \%$ in correctly detecting fall conditions and 95\% specificity in avoiding false alarms during some daily activities. On the one hand, these results depend on presetting of the accelerometer thresholds, but, on the other hand, it indicates possible adjustment (preset) to suit different holders' conditions. This would be a prospective research under the supervision of physicians.

Finally, the Internet Cloud access allows for management of all daily data about the AD's health conditions (ie, data from the three biomarkers). This data can be saved, checked, and managed (eg, printed or deleted) by a medical care provider. The microcontroller generates alarms to AD patient in case of medical condition associated to the three biomarkers and the fall down condition. Although there is no SOS button, which can be added, the microcontroller can be programmed to produce "loud" recordings to the surrounding people when $\mathrm{AD}$ patients encountered abnormal biomarkers or fall down conditions.

There are reports in literature evaluating various solutions for fall detection accidents. They always attempted to discern falls from common ADL activities. However, the solutions vary in certain aspects such as the type of sensor and the sensor placement. Table 5 illustrates examples from these reports. Some reports utilized micro-electro-mechanical systems ${ }^{31}$ (MEMS) or accelerometer only, ${ }^{27,30}$ while others used the accelerometer being integrated to gyroscope with/without magnetometer. ${ }^{26,29,33}$ Some researchers preferred to collaborate the accelerometer with Heart rate Variability (HRV) sensor from the ECG signal (ie, stress indicator) ${ }^{28}$ or infra-red sensor (ie, position indicator). ${ }^{31}$ Other researchers utilized artificial intelligence technology (eg, machine learning program) based on acceleration measurements during fall accidents. ${ }^{32}$ On the other hand, there is also no agreement of the place where to put 
Table 5 Fall Detection Systems Using Gyroscope/Accelerometer

\begin{tabular}{|c|c|c|c|c|c|}
\hline \multirow{2}{*}{$\begin{array}{l}\text { Reference } \\
\text { Number }\end{array}$} & \multirow{2}{*}{$\begin{array}{c}\text { No. of } \\
\text { Volunteers }\end{array}$} & \multirow[t]{2}{*}{ Type of Sensor } & \multirow[t]{2}{*}{ Sensor Placement } & \multicolumn{2}{|c|}{ Observed Performance } \\
\hline & & & & Sensitivity & Specificity \\
\hline [26] [2009] & $\begin{array}{l}3 \text { Healthy } \\
\text { subjects }\end{array}$ & Accelerometer and gyroscope & Chest, Thigh & $91 \%$ & $92 \%$ \\
\hline [27] [20।5] & & Accelerometer & Waist & $97 \%$ & $98.3 \%$ \\
\hline [28] [20।5] & $\begin{array}{l}50 \text { Healthy } \\
\text { subjects }\end{array}$ & Accelerometer \& HRV sensor & Chest & \multicolumn{2}{|c|}{ Accuracy $96 \%$ to $100 \%$} \\
\hline [29] [20।7] & $\begin{array}{l}\text { I5 Healthy } \\
\text { subjects }\end{array}$ & $\begin{array}{c}\text { Accelerometer, Gyroscope, and } \\
\text { magnetometer }\end{array}$ & Shoulder, waist, foot & $100 \%$ & $\begin{array}{c}91.1 \% \text { at shoulder, } \\
100 \% \text { at waist } \\
78.5 \% \text { at foot }\end{array}$ \\
\hline [30] [20।7] & $\begin{array}{l}10 \text { Healthy } \\
\text { subjects }\end{array}$ & G-Force from accelerometer & Smartphone (pocket in pants) & $83 \%$ & $92 \%$ \\
\hline$[3 \mid][2017]$ & $\begin{array}{l}20 \text { Healthy } \\
\text { subjects }\end{array}$ & MEMS and IR signal & Waist & $98 \%$ & $93 \%$ \\
\hline [32] [20|4] & $\begin{array}{l}\text { I4 Healthy } \\
\text { subjects }\end{array}$ & $\begin{array}{c}\text { Accelerometer, Gyroscope, and } \\
\text { magnetometer + Machine Learning }\end{array}$ & $\begin{array}{l}\text { Head, chest, waist, right wrist, } \\
\text { right thigh, and right ankle }\end{array}$ & $99 \%$ & $99 \%$ \\
\hline [33] [2017] & $\begin{array}{l}20 \text { Healthy } \\
\text { subjects }\end{array}$ & Accelerometer and gyroscope & Vest & $99 \%$ & $95 \%$ \\
\hline This work & $\begin{array}{l}\text { I3 Healthy } \\
\text { subjects }\end{array}$ & Accelerometer and gyroscope & Wrist & $93 \%$ & $95 \%$ \\
\hline
\end{tabular}

the fall detection device. The sensor was stationed at either head $^{32}$ chest, $^{26,32}$ thigh, ${ }^{26,32}$ waist, ${ }^{27,29,31,32}$ shoulder, $^{29}$ wrist, $^{32}$ or foot. ${ }^{29}$ Some researchers preferred the smart phone style so the fall detection device can be put in the pocket of pants, ${ }^{30}$ while other researchers preferred to design a dedicated vest. ${ }^{33}$ Table 5 demonstrates that there is no evidence of agreement between researchers groups. Most of reports in literature employs different types and brand names of accelerometers. This indicates that they had used different 3D acceleration thresholds. However, they all reported outstanding sensitivity and specificity values higher than $90 \%$ and may reach $100 \%$. These records were obtained from healthy subjects, who were asked to repeat simulations of either fall detection or ADL activities. Therefore, Table 5 shows that the suggested SWDM yielded close results to other reports in literature. In this paper, however, the SWDM is a multifunction prototype put on the wrist only. We believe that, as in references no. ${ }^{1}$ placing many fall detection devices at various parts of the body would burden the holders. The SWDM was applied on healthy people yielding comparative sensitivity and specificity results. It was difficult, as in reports in Table 5, to test the prototype on abnormal or elderly subjects asking them to follow simulations of falling down accidents. The
SWDM presents an initial results toward designing device for elderly people that on one hand detect fall accidents and on the other hand observe multiple biomarker (not only the HRV) via the GPS and WiFi microcontroller with on-line Internet Cloud access.

The future work of this paper would be to minimize the dimension of the suggested prototype, so it becomes more comfortable. This may be achieved by using a smaller electronics such as micro or nano-embedded systems). Also, there is a need to improve fall down detection accuracy (sensitivity and specificity). There is a need to test the SWDM on elderly or AD patients with different ages and different disease stages, but this should require medical precautions because it implies falling down tests and risks.

\section{Conclusion}

The aim of this study is to develop an assistive wearable prototype that can be used as a solution to locate the Alzheimer's patient while also monitoring other vital parameters such as temperature, heart rate, and oxygen level. The prototype can also send an alert if the patient suddenly collapses due to any health complications. It incorporates a Wi-Fi microcontroller that allows saving daily information as well as 
monitoring the GPS location of the AD patient. The system was checked on 13 volunteers, yielding 93\% sensitivity in correctly detecting fall down conditions, and 95\% specificity in avoiding false alarms. The Wi-Fi microcontroller, GPS, biometrics acquisition, and the fall down alert circuits operated successfully. The prototype is as a trustworthy device for daily life support for AD patients.

\section{Acknowledgments}

The authors are very thankful for the staff of biomedical engineering department and participants that contributed in/for the objective of this study.

\section{Funding}

The authors did not receive any financial support to conduct this research.

\section{Disclosure}

The authors report no conflicts of interest in this work.

\section{References}

1. World Parkinson Coalition. Abstract book of the 5th World Parkinson Congress, Kyoto, Japan, June 4-7, 2019. J Parkinsons Dis. 2019;9 (s1):1-278. doi:10.3233/jpd-199900

2. Wang T. Acupuncture for Brain.Springer, Cham; 2021. doi:10.1007/ 978-3-030-54666-3

3. Reisberg B, Prichep L, Mosconi L, et al. The pre-mild cognitive impairment, subjective cognitive impairment stage of Alzheimer's disease. Alzheimers Dement. 2008;4(1 SUPPL. 1). doi:10.1016/j. jalz.2007.11.017

4. Kourtis LC, Regele OB, Wright JM, Jones GB. Digital biomarkers for Alzheimer's disease: the mobile/wearable devices opportunity. Npj Digit Med. 2019;2(1). doi:10.1038/s41746-019-0084-2

5. Eshkoor SA, Hamid TA, Nudin SS, Adiah H, Mun CY. Association between dentures and the rate of falls in dementia. Med Devices Evid Res. 2014;7(1). doi:10.2147/MDER.S63220

6. Srivastava S, Ahmad R, Khare SK. Alzheimer's disease and its treatment by different approaches: a review. Eur J Med Chem. 2021;216. doi:10.1016/j.ejmech.2021.113320

7. Guarino A, Favieri F, Boncompagni I, Agostini F, Cantone M, Casagrande M. Executive functions in Alzheimer disease: a systematic review. Front Aging Neurosci. 2019;10. doi:10.3389/ fnagi.2018.00437

8. Nogueira MML, Neto JPS, Dourado MCN. Quality of life of people with Alzheimer disease: comparison between Dyads Degree of Kinship. J Geriatr Psychiatry Neurol. 2021;34(2):119-127. doi:10.1177/0891988720915521

9. Furht B, Agarwal A. Handbook of Medical and Healthcare Technologies. New York, NY: Springer; 2013. doi:10.1007/9781-4614-8495-0

10. Chen S, Jin X, Zhang L, Wan J. A comprehensive review of IoT technologies and applications for healthcare. Communications in Computer and Information Science. Vol. 1424; 2021. doi:10.1007/ 978-3-030-78621-2_29.
11. Abbate S, Avvenuti M, Light J. MIMS: a minimally invasive monitoring sensor platform. IEEE Sens J. 2012;12(3):677-684. doi:10.1109/JSEN.2011.2149515

12. Abbate S, Avvenuti M, Light J. Usability study of a wireless monitoring system among Alzheimer's disease elderly population. Int J Telemed Appl. 2014;2014. doi:10.1155/2014/617495

13. Caring.com. Freedom Guardian Watch Review; 2021. Available from: https://www.caring.com/best-medical-alert-systems/freedomguardian-watch/. Accessed July 14, 2021.

14. SafeTracks GPS Canada Inc. GPS-SmartSole ${ }^{\text {TM; }}$ 2021. Available from: https://www.iloctech.com/. Accessed November 23, 2021.

15. Mehrabian S, Extra J, Wu YH, Pino M, Traykov L, Rigaud AS. The perceptions of cognitively impaired patients and their caregivers of a home telecare system. Med Devices Evid Res. 2014;8. doi:10.2147/MDER. S70520

16. Boppana L, Kumari P, Chidrewar R, Gadde PK. Smart cap for Alzheimer patients using deep learning. IEEE Region 10 Annual International Conference, Proceedings/TENCON. Vol. 2019; October, 2019. doi:10.1109/TENCON.2019.8929412.

17. Lee G, Nho K, Kang B, et al. Predicting Alzheimer's disease progression using multi-modal deep learning approach. Sci Rep. 2019;9 (1). doi:10.1038/s41598-018-37769-z

18. AnalogDevices. Pulse oximeter and heart-rate sensor IC for wearable health; 2014. Available from: https://www.maximintegrated.com/en/pro ducts/sensors/MAX30100.html\#tech-docs. Accessed November 17, 2021.

19. Altman DG, Bland JM. Assessing agreement between methods of measurement. Clin Chem. 2017;63(10):1653-1654. doi:10.1373/ clinchem.2016.268870

20. Wojtusiak J, Mogharab Nia R. Location prediction using GPS trackers: can machine learning help locate the missing people with dementia? Internet Things. 2021;13:100035. doi:10.1016/j.iot.2019.01.002

21. Bal D, Islam Tusher MM, Rahman M, Rahman Saymon MS. NAVIX: a wearable navigation system for visually impaired persons. 2020 2nd International Conference on Sustainable Technologies for Industry 4.0, STI 2020; 2020. doi:10.1109/STI50764.2020.9350480.

22. Daum CH, Neubauer N, Oliva C, Beleno R, Liu L. Accuracy and usability of a mobile alert system for community citizens to locate persons with dementia who get lost. Innov Aging. 2019;3 (Supplement_1):S453-S454. doi:10.1093/geroni/igz038.1698

23. Aljehani SS, Alhazmi RA, Aloufi SS, Aljehani BD, Abdulrahman R. ICare: applying IoT technology for monitoring Alzheimer's patients. 1st International Conference on Computer Applications and Information Security, ICCAIS 2018; 2018. doi:10.1109/ CAIS.2018.8442010.

24. Anzalone P. Equivalence of earlobe site blood glucose testing with finger stick. Clin Nurs Res. 2008;17(4):251-261. doi:10.1177/ 1054773808325050

25. Ramachandran A, Karuppiah A. A survey on recent advances in wearable fall detection systems. Biomed Res Int. 2020;2020:2167160. doi:10.1155/2020/2167160

26. Li Q, Stankovic JA, Hanson MA, Barth AT, Lach J, Zhou G. Accurate, fast fall detection using gyroscopes and accelerometer-derived posture information. Proceedings - 2009 6th International Workshop on Wearable and Implantable Body Sensor Networks, BSN 2009; 2009. doi:10.1109/BSN.2009.46.

27. Wu F, Zhao H, Zhao Y, Zhong H. Development of a wearable-sensorbased fall detection system. Int J Telemed Appl. 2015;2015. doi:10.1155/2015/576364

28. Shahiduzzaman M. Fall detection by accelerometer and heart rate variability measurement. Glob J Comput Sci Technol. 2015;15(3-G).

29. Mao A, Ma X, He Y, Luo J. Highly portable, sensor-based system for human fall monitoring. Sensors (Switzerland). 2017;17(9):2096. doi:10.3390/s17092096 
30. Chaitep T, Chawachat J. A 3-Phase threshold algorithm for smartphone-based fall detection. ECTI-CON 2017-2017 14th International Conference on Electrical Engineering/Electronics, Computer, Telecommunications and Information Technology; 2017. doi:10.1109/ECTICon.2017.8096203.

31. Chen J, Kwong K, Chang D, Luk J, Bajcsy R. Wearable sensors for reliable fall detection. Annual International Conference of the IEEE Engineering in Medicine and Biology - Proceedings. Vol. 7; 2005. doi:10.1109/iembs.2005.1617246.
32. Özdemir AT, Barshan B. Detecting falls with wearable sensors using machine learning techniques. Sensors (Switzerland). 2014;14 (6):10691-10708. doi:10.3390/s140610691

33. He J, Bai S, Wang X. An unobtrusive fall detection and alerting system based on Kalman Filter and Bayes Network Classifier. Sensors (Switzerland). 2017;17(6):1393. doi:10.3390/s17061393
Medical Devices: Evidence and Research

\section{Publish your work in this journal}

Medical Devices: Evidence and Research is an international, peerreviewed, open access journal that focuses on the evidence, technology, research, and expert opinion supporting the use and application of medical devices in the diagnosis, monitoring, treatment and management of clinical conditions and physiological processes. The identification of novel devices and optimal use of existing devices
Dovepress

which will lead to improved clinical outcomes and more effective patient management and safety is a key feature of the journal. The manuscript management system is completely online and includes a very quick and fair peer-review system. Visit http:// www.dovepress.com/testimonials.php to read real quotes from published authors. 